\title{
Addition of Amide Proton Transfer Imaging to FDG-PET/CT Improves Diagnostic Accuracy in Glioma Grading: A Preliminary Study Using the Continuous Net Reclassification Analysis
}

\author{
(D) A. Sakata, (D) T. Okada, (D) Y. Yamamoto, (D). Fushimi, (D)T. Dodo, DY. Arakawa, (D). Mineharu, (D) B. Schmitt, (D). Miyamoto, and
} (D) K. Togashi

\begin{abstract}
BACKGROUND AND PURPOSE: Amide proton transfer imaging has been successfully applied to brain tumors, however, the relationships between amide proton transfer and other quantitative imaging values have yet to be investigated. The aim was to examine the additive value of amide proton transfer imaging alongside $\left[{ }^{18} \mathrm{~F}\right]$ FDG-PET and DWI for preoperative grading of gliomas.
\end{abstract}

MATERIALS AND METHODS: Forty-nine patients with newly diagnosed gliomas were included in this retrospective study. All patients had undergone MR imaging, including DWI and amide proton transfer imaging on 3T scanners, and $\left[{ }^{18} \mathrm{~F}\right] \mathrm{FDG}-\mathrm{PET}$. Logistic regression analyses were conducted to examine the relationship between each imaging parameter and the presence of high-grade (grade III and/or IV) glioma. These parameters included the tumor-to-normal ratio of FDG uptake, minimum ADC, mean amide proton transfer value, and their combinations. In each model, the overall discriminative power for the detection of high-grade glioma was assessed with receiver operating characteristic curve analysis. Additive information from minimum ADC and mean amide proton transfer was also evaluated by continuous net reclassification improvement. $P<.05$ was considered significant.

RESULTS: Tumor-to-normal ratio, minimum ADC, and mean amide proton transfer demonstrated comparable diagnostic accuracy in differentiating high-grade from low-grade gliomas. When mean amide proton transfer was combined with the tumor-to-normal ratio, the continuous net reclassification improvement was $0.64(95 \% \mathrm{Cl}, 0.036-1.24 ; P=.04)$ for diagnosing high-grade glioma and 0.95 (95\% $\mathrm{Cl}$, $0.39-1.52 ; P=.001)$ for diagnosing glioblastoma. When minimum ADC was combined with the tumor-to-normal ratio, the continuous net reclassification improvement was $0.43(95 \% \mathrm{Cl},-0.17-1.04 ; P=.16)$ for diagnosing high-grade glioma, and $1.36(95 \% \mathrm{Cl}, 0.79-1.92 ; P<.001)$ for diagnosing glioblastoma.

CONCLUSIONS: Addition of amide proton transfer imaging to FDG-PET/CT may improve the ability to differentiate high-grade from low-grade gliomas.

ABBREVIATIONS: $A D C_{\min }=$ minimum $A D C ; A P T=$ amide proton transfer; $A U C=$ area under the curve; $\mathrm{NRI}=$ net reclassification improvement; $R O C=$ receiver operating characteristic; $\mathrm{SO}$ image $=$ reference dataset acquired without presaturation; SUV $=$ standard uptake value; $\mathrm{T} / \mathrm{N}=$ tumor-to-normal

M $\mathrm{R}$ imaging has an established role for the localization, characterization, and diagnosis of brain tumors, as well as for assessing the effects of treatment. Several studies have demonstrated the utility of various types of advanced sequences for grading brain tumors through the visualization of water diffusion,

Received May 19, 2017; accepted after revision October 20.

From the Department of Diagnostic Imaging and Nuclear Medicine (A.S., T.O., Y.F., T.D., K.T.), Brain Research Center (T.O.), Department of Neurosurgery (Y.A., Y.M., S.M.), and Department of Healthcare Epidemiology (Y.Y.), School of Public Health, Kyoto University Graduate School of Medicine, Kyoto, Japan; and Magnetic Resonance (B.S.), Siemens Healthcare, Bayswater, Australia.

This work was supported by a Grant-in-Aid for Scientific Research on Innovative Areas "Initiative for High-Dimensional Data-Driven Science through Deepening of Sparse Modeling" of Ministry of Education, Culture, Sports, Science and Technology, Japan, provided to K.T. (MEXT grant number 25120002).

Please address correspondence to T. Okada, MD, Human Brain Research Center, Kyoto University Graduate School of Medicine, 54 Shogoin Kawaharacho, Sakyoku, Kyoto 606-8507, Japan; e-mail: tomokada@kuhp.kyoto-u.ac.jp

tumor metabolites, or perfusion characteristics. ${ }^{1,2}$ For pre- and postoperative assessment of gliomas, DWI has been the most commonly used of these advanced sequences, ${ }^{2}$ and the derived $\mathrm{ADC}$ is a quantitative parameter that is inversely correlated with tumor cellularity and hence glioma grade. ${ }^{3,4}$ However, its clinical impact has remained limited because of the substantial overlap in regional ADCs among gliomas of different grades. ${ }^{5}$

PET is another quantitative imaging technique used in neurooncology. ${ }^{2}$ The standard uptake value (SUV) obtained with FDGPET also plays an important role in the grading of brain tumors. ${ }^{2}$ High-grade gliomas generally show a higher level of glucose metabolism than low-grade gliomas and therefore exhibit increased

\footnotetext{
--.- Indicates open access to non-subscribers at www.ajnr.org

$\triangle \Delta$ Indicates article with supplemental on-line photos.

http://dx.doi.org/10.3174/ajnr.A5503
}

AJNR Am J Neuroradiol 39:265-72 Feb 2018 www.ajnr.org 
SUV. However, physiologic FDG uptake by the brain may obscure tumor uptake. PET also has shortcomings in terms of the cost, exposure to radiation, and relatively low spatial resolution.

In addition to the aforementioned methods, chemical exchange-dependent saturation transfer imaging has recently emerged as a new contrast mechanism for MR imaging in the field of cellular and molecular imaging. ${ }^{6-8}$ This method of magnetization transfer imaging has several variants, one of which is amide proton transfer (APT) imaging, which focuses on endogenous cytosolic proteins and peptides with amide protons in the peptide bond. ${ }^{9}$ This technique has been successfully applied to human brain tumors. ${ }^{3,10-16}$ Some reports have shown that the APT asymmetry value is useful in tumor grading, allowing differentiation of pseudoprogression from recurrence ${ }^{17}$ and the assessment of treatment response. ${ }^{18,19}$ However, the relationship between APT and other quantitative imaging values has yet to be investigated.

The purposes of this study were the following: 1) to compare the diagnostic accuracy of APT imaging for preoperative grading of glial tumors with that of DWI and $\left[{ }^{18} \mathrm{~F}\right] \mathrm{FDG}-\mathrm{PET}$, and 2) to examine the additive value of APT imaging combined with $\left[{ }^{18} \mathrm{~F}\right]$ FDG-PET and DWI for the preoperative grading of gliomas. To quantify the additive value of APT imaging, we used a statistical method called the net reclassification index (NRI), an index that shows how well a new model reclassifies subjects. ${ }^{20} \mathrm{NRI}$ is calculated as the difference in the proportion of subjects classified correctly as opposed to wrongly classified after application of a new model. This measure can demonstrate the superiority of a new model over a previous one.

\section{MATERIALS AND METHODS}

Our institutional review board approved this retrospective study (R0120), and the requirement to obtain informed consent was waived.

\section{Patients}

Eighty-three consecutive adult (older than 18 years of age) patients with suspected supratentorial gliomas who were treated at our hospital between December 2012 and April 2015 were reviewed. The inclusion criteria were the following: 1) pathologic diagnosis of grades II-IV diffuse glioma (2007 World Health Organization criteria $)^{21}$; 2) the availability of results from preoperative MR imaging, including DWI and APT imaging, and FDGPET obtained within the year before the operation. Twenty-four patients did not meet the inclusion criteria because of no histology or histology other than glioma $(n=7)$ or incomplete datasets $(n=17)$. Ten patients were also excluded because of major therapeutic intervention (such as an operation, radiation therapy, or chemotherapy including steroids) before imaging $(n=5)$ or severe artifacts $(n=5)$. Finally, we analyzed data from 49 patients who underwent $\left[{ }^{18} \mathrm{~F}\right]$ FDG-PET/CT and MR imaging, including DWI and APT imaging. Subsets from this patient population $(n=$ 26; 13 each) have been used in previous publications, ${ }^{15,22}$ though not with the research focus presented in the current study (Fig 1).

\section{Imaging Acquisition}

DWI. MR imaging was conducted by using two 3T scanners (Magnetom Trio; Siemens, Erlangen, Germany) with 32-channel head coils. In addition to the conventional FLAIR sequence

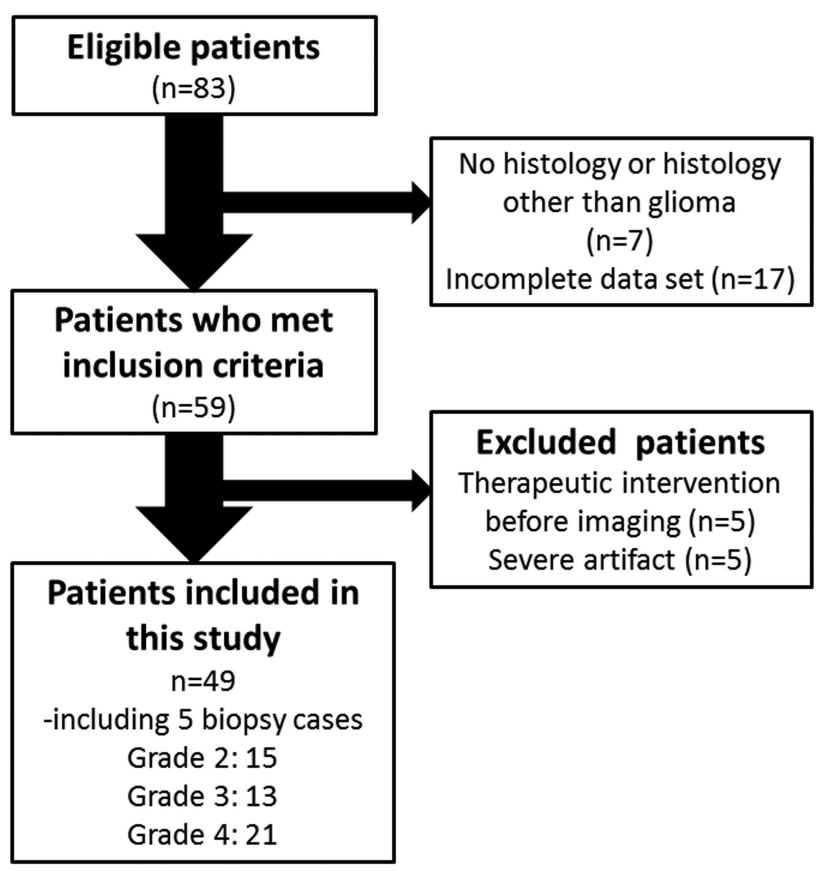

FIG 1. Flowchart showing the 83 eligible patients who received a histologic diagnosis of primary glioma after MR imaging and PET and subsequently underwent an operation during the 29-month period.

(TR/TE, 12,000/100 ms; TI, $2760 \mathrm{~ms}$; flip angle, $120^{\circ}$; resolution, $0.69 \times 0.69 \mathrm{~mm})$, T2-weighted FSE (TR/TE, 3200/79 ms; flip angle, $120^{\circ}$; resolution, $\left.0.49 \times 0.49 \mathrm{~mm}\right)$ and pre- and postcontrast-enhanced T1-weighted imaging were acquired (using magnetization-prepared rapid acquisition of gradient echo with the following settings: TR/TE, $6 / 2.26 \mathrm{~ms}$; flip angle, $15^{\circ}$; resolution, $0.9 \times 0.9 \times 0.9 \mathrm{~mm})$. Contrast materials used were $0.1 \mathrm{mmol} / \mathrm{kg}$ of gadopentetate dimeglumine (Magnevist; Bayer HealthCare Pharmaceuticals, Wayne, New Jersey) or gadoteridol (ProHance; Bracco Diagnostics, Princeton, New Jersey). DWI (TR/TE, 5000/77 ms; resolution, $1.4 \times 1.4 \mathrm{~mm}$; slice thickness, $3 \mathrm{~mm}$ with a 1-mm gap) was performed with motion-probing gradients of $b=1000 \mathrm{~s} / \mathrm{mm}^{2}$ applied in 3 orthogonal directions. Images without motion-probing gradients were also obtained, and ADC maps were calculated.

APT. APT imaging was conducted using a prototype $3 \mathrm{D}$ gradient-echo pulse sequence (TR/TE, 8.3/3.3 ms; flip angle, $12^{\circ} ; 24$ slices; resolution, $1.72 \times 1.72 \times 4 \mathrm{~mm})$. The presaturation pulses consisted of 3 consecutive radiofrequency pulses of 100 -ms duration with 100-ms interpulse delays and a time-average amplitude of $2 \mu \mathrm{T}$. Eighteen consecutive datasets were acquired with different offset frequencies $\Delta \omega(0, \pm 0.6, \pm 1.2, \pm 1.8, \pm 2.4, \pm 3.0, \pm 3.6$, \pm 4.2 , and $\pm 4.8 \mathrm{ppm}$ ) from the bulk water resonance. Saturated images $(\mathrm{S}[\Delta \omega])$ were normalized with a reference dataset acquired without presaturation (S0 image). The APT effect was calculated as the asymmetry of the magnetization transfer rate using the following equation: $A P T_{\text {asym }}=(S[-3.5 \mathrm{ppm}]-S[+3.5$ $\mathrm{ppm}]) / S O \times 100(\%)$. The $A P T_{\mathrm{asym}}$ at $3.5 \mathrm{ppm}$ was obtained from linear interpolation between the originally sampled points using an offset resolution of $0.1 \mathrm{ppm}$ and subsequent correction for inhomogeneity of the static magnetic field, as previously described. ${ }^{23}$ 
$\left[{ }^{18} \mathrm{~F}\right]$ FDG-PET. PET was performed with a PET/CT scanner (Discovery ST Elite; GE Healthcare, Milwaukee, Wisconsin). Each patient fasted for at least 4 hours before PET. After intravenous administration of FDG at $4 \mathrm{MBq} / \mathrm{kg}$ body weight, the patient rested in a waiting room for 30 minutes. After performing lowdose CT for attenuation correction, we performed emission scans of the brain for 15 minutes with a $128 \times 128$ matrix and 47 slices (resolution, $2.0 \times 2.0 \times 3.27 \mathrm{~mm}$ ). The reconstructed PET data were converted to SUV data using the following equation: $\mathrm{SUV}=$ Count at a Pixel $\left(\mathrm{kBq} / \mathrm{cm}^{3}\right) /$ Injection Dose $(\mathrm{MBq}) /$ Weight $(\mathrm{kg})$.

\section{Imaging Analysis}

All image processing was conducted by 2 neuroradiologists (A.S. and T.O., with 4 and 22 years of experience of imaging processing) in consensus to double-check the quality. Images were coregistered using SPM8 software (http://www.fil.ion.ucl.ac.uk/spm/ software/spm12) implemented in Matlab (MathWorks, Natick, Massachusetts). All postcontrast T1-weighted, ADC, S0, and APT images were coregistered to the corresponding FLAIR images and resliced; S0 images were used for coregistration of APT images to anatomic images. Registrations were visually inspected and manually corrected if necessary. FDG-PET images were not coregistered to MR images because the image resolution and contrast were different.

\section{Minimum ADC}

Two board-certified neuroradiologists (A.S. and T.D., each with 7 years of experience in diagnostic neuroradiology) analyzed the ADC maps independently using ImageJ, Version 1.48 (National Institutes of Health, Bethesda, Maryland). All continuous sections that included tumor were evaluated by placing 3 circular ROIs over the low-intensity area corresponding to the solid portion of the tumor. ${ }^{24}$ The area of the ROIs was predefined as 30 $\mathrm{mm}^{2}$, and each ROI was carefully positioned to avoid contamination from adjacent tissues and estimation errors caused by necrosis, hemorrhage, or calcification.

\section{APT $_{\text {mean }}$}

For the APT images, board-certified neuroradiologists (A.S. and T.D.) independently placed an ROI over a representative slice of the tumor (1 ROI per patient). In case of tumors with an enhancing portion, ROIs were drawn on the enhanced area (viable tumor core) on the contrast-enhanced T1-weighted images. When such enhancement was absent, ROIs were drawn by selecting abnormal signal areas on the FLAIR images. Foci of necrosis, hemorrhage, or calcification were manually avoided. All ROIs were applied to the resliced $\mathrm{APT}$ images, and mean values $\left(\mathrm{APT}_{\text {mean }}\right)$ were calculated.

\section{Tumor-to-Normal Ratio}

For SUV $\mathrm{S}_{\text {maximum }}$ measurement, 2 neuroradiologists with board certification in nuclear medicine (Y.F. and T.O., with 13 and 18 years of experience respectively) independently drew several oval ROIs (diameter $=10 \mathrm{~mm}$ ) to include the area with the highest SUV. The number of ROIs varied, depending on the size of the tumor (median, 9; range, 1-25). Fifteen ROIs (5 ROIs on each of 3 axial slices) were also placed on the contralateral frontoparietal gray matter. When a tumor occupied the bilateral lobes, the hemi- sphere with the largest part of the tumor was defined as the side of the tumor. Semiquantitative analysis was performed using the tumor-to-normal $(\mathrm{T} / \mathrm{N})$ ratio, defined as the $\mathrm{SUV}_{\text {maximum }}$ in the tumor divided by the average SUV of the normal gray matter. ${ }^{25}$

\section{Pathologic Analysis}

Tumors were graded according to the 2007 World Health Organization classification of brain tumors by board-certified neuropathologists with $>15$ years of experience. ${ }^{21}$ The grade of glial tumor was determined on the basis of histologic characteristics such as nuclear atypia, mitosis, microvascular proliferation, and the presence of necrosis. Immunohistochemical analyses were used when necessary. Grade III and IV tumors were considered high-grade, and grade II tumors, as low-grade.

\section{Statistical Analysis}

To determine the interrater reliability for continuous data $(\mathrm{T} / \mathrm{N}$ ratio, minimum $\mathrm{ADC}\left[\mathrm{ADC}_{\text {min }}\right]$ and $\mathrm{APT}_{\text {mean }}$ measurements), the intraclass correlation coefficient was calculated with a 2-way random model with absolute agreement on average measures. Interpretation of the intraclass correlation coefficient followed methods described by Landis and $\mathrm{Koch}^{26}:<0$, no reproducibility; $0.0-0.20$, slight reproducibility; $0.21-0.40$, fair reproducibility; $0.41-0.60$, moderate reproducibility; $0.61-0.80$, substantial reproducibility; and 0.81-1.00, almost perfect reproducibility.

To assess the ability to correctly differentiate high-grade gliomas, we conducted receiver operating characteristic (ROC) curve analysis for $\mathrm{APT}_{\text {mean }}, \mathrm{ADC}_{\text {min }}$, and the $\mathrm{T} / \mathrm{N}$ ratio. We compared the areas under the curve (AUCs) using the method described by DeLong et al. ${ }^{27}$

Clinical models were created for logistic regression analysis, combining 2 of the 3 parameters $\mathrm{APT}_{\text {mean }}, \mathrm{ADC}_{\text {min }}$, and the $\mathrm{T} / \mathrm{N}$ ratio. The added value of the additional imaging beyond $\left[{ }^{18} \mathrm{~F}\right]$ FDG-PET (which showed the highest AUC for the primary outcome) was quantified by consecutively extending the basic model and assessing the increase in AUC. Furthermore, the number of patients correctly reclassified after adding these parameters was expressed as the NRI. The continuous NRI generalizes a summary measure proposed for reclassification tables by eliminating risk categories and defining any increase in model-based probability resulting from the addition of a new marker as upward reclassification, and any decrease as downward reclassification. The continuous NRI index is equal to twice the difference in the probabilities of upward reclassification for the events minus the nonevents. ${ }^{20}$ Internal validation for both logistic regression analysis and NRI was performed with 1000 bootstrapped samples. Furthermore, we conducted additional ROC and NRI analyses to evaluate the additive value of APT to ADC.

Statistical analysis was performed using STATA, Version 13 software (StataCorp, College Station, Texas). $P<.05$ was considered indicative of a significant difference.

\section{RESULTS}

\section{Patient Characteristics}

Forty-nine patients (32 men, 17 women) with a new histopathologic diagnosis of glioma and adequate image sets were included in this study. The mean age was 58.3 years (range, 21-90 years). 
Grade II glioma was seen in 15 patients ( 9 diffuse astrocytomas, 4 oligodendrogliomas, 2 oligoastrocytomas); grade III glioma, in 13 patients ( 9 anaplastic astrocytomas, 1 anaplastic oligodendroglioma, 3 anaplastic oligoastrocytomas); and glioblastoma, in 21 patients. Five patients underwent surgical biopsy, and 44 patients underwent surgical resection. The characteristics of the patients with low- and high-grade gliomas are given in Table 1. Representative cases are shown in Figs 2 and 3.

\section{Interrater Reliability}

Interrater reliability showed almost perfect reproducibility for the $\mathrm{T} / \mathrm{N}$ ratio, $\mathrm{ADC}_{\text {min }}$, and $\mathrm{APT}$ measurements, with intraclass

Table 1: Patient characteristics

\begin{tabular}{lcc}
\hline & $\begin{array}{c}\text { Low-Grade } \\
\text { Gliomas }\end{array}$ & $\begin{array}{c}\text { High-Grade } \\
\text { Gliomas }\end{array}$ \\
\hline Patients (No.) & 15 & 34 \\
$\mathrm{M} / \mathrm{F}$ & $10 / 5$ & $22 / 12$ \\
Age (yr) & $51.5 \pm 15.9$ & $59.5 \pm 15.6$ \\
$\quad \begin{array}{l}\text { Median (range) of interval between } \\
\quad \text { surgery and MRI (day) }\end{array}$ & $45(1-168)$ & $13(0-76)$ \\
$\quad \begin{array}{l}\text { Median (range) of interval between } \\
\quad \text { surgery and PET (day) }\end{array}$ & $51(15-306)$ & $13(1-218)$ \\
$\mathrm{T}_{\mathrm{N}}$ & $0.75 \pm 0.26$ & $1.19 \pm 0.43$ \\
$\mathrm{ADC}_{\text {min }}\left(\times 10^{-6} \mathrm{~mm}^{2} / \mathrm{s}\right)$ & $980 \pm 179$ & $757 \pm 221$ \\
$\mathrm{APT}_{\text {mean }}(\%)$ & $0.87 \pm 0.39$ & $1.33 \pm 0.46$ \\
\hline
\end{tabular}

correlation coefficients of 0.89 (95\% confidence interval, 0.810.94 ) for $\mathrm{T} / \mathrm{N}$ ratio, 0.90 (95\% CI, 0.82-0.95) for $\mathrm{ADC}_{\mathrm{min}}$, and 0.97 (95\% CI, 0.95-0.99) for APT. Given the high interrater reliability, the subsequent statistical evaluation of these measurements used the mean of the values measured by both raters for each patient.

\section{ROC Curve for Each Single Method and Comparison of AUCs}

Table 2 and On-line Fig 1 summarize the results of ROC curve analysis for each parameter. No significant differences were seen among $\mathrm{T} / \mathrm{N}_{\text {ratio, }} \mathrm{APT}_{\text {mean }}$, and $\mathrm{ADC}_{\text {min }}$ in the differentiation of higher grade gliomas from lower grade ones (grades III and IV versus grade II, $P=.60$; grade IV versus grades II and III, $P=.68$ ).

\section{Logistic Regression Analysis to Evaluate the Added Value of APT Imaging to [ ${ }^{18}$ F] FDG-PET for Differentiation of High- from Low-Grade Gliomas}

Table 3 and On-line Fig 2 summarize the results for the AUCs of each combination of the 2 parameters. In comparison with the AUC for the $\mathrm{T} / \mathrm{N}$ ratio alone, some tendencies toward improvement were seen with either combination of the $\mathrm{T} / \mathrm{N}$ ratio and $\mathrm{APT}_{\text {mean }}$, but the differences did not reach statistical significance.

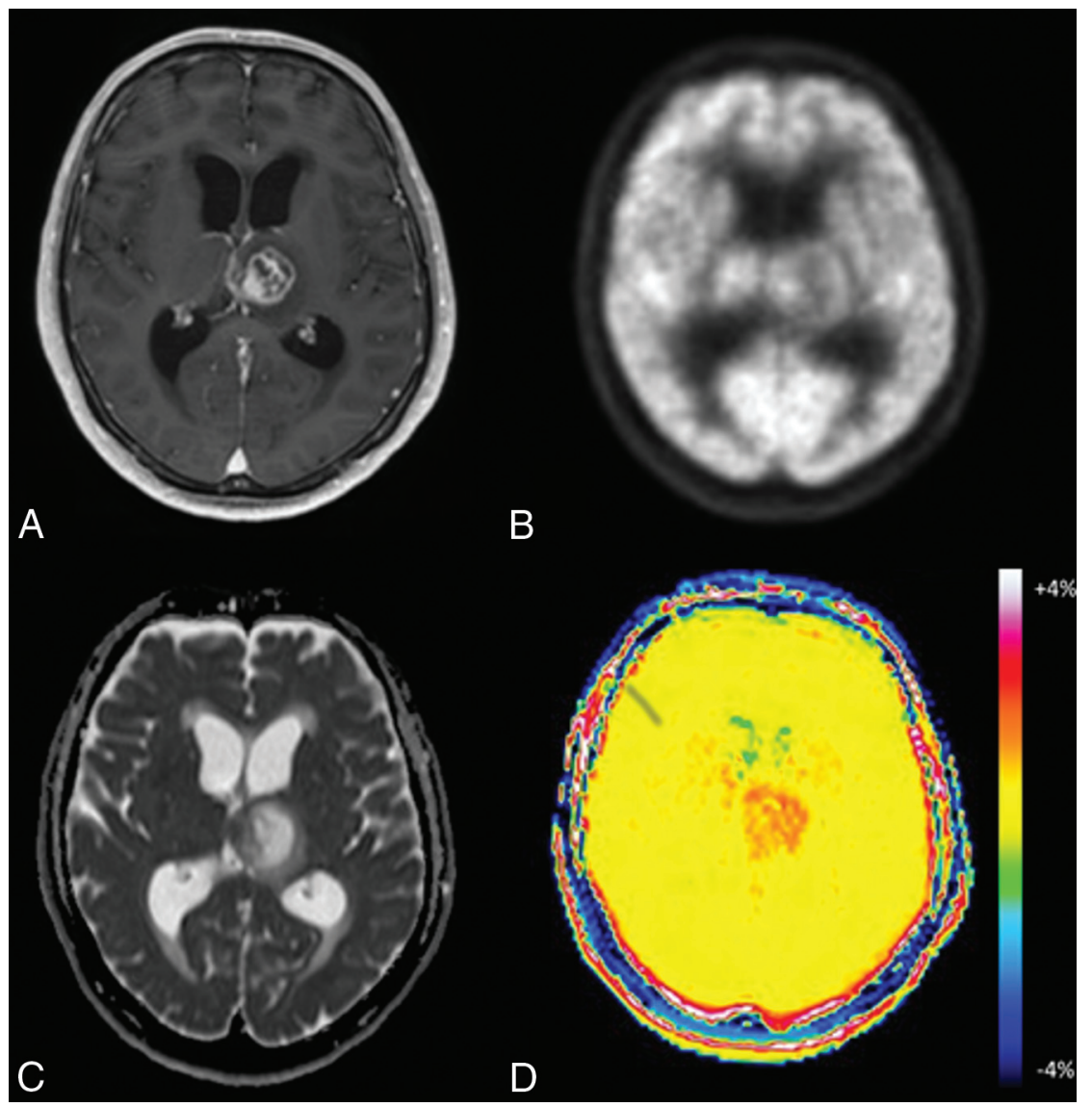

FIG 2. Glioblastoma in a 65-year-old man. $A$, Axial MR imaging shows a contrast-enhancing lesion in the left thalamus. $B$, FDG-PET shows less uptake by the lesion compared with gray matter. $C$, On the ADC map, the medial portion of the tumor demonstrates focal low-to-intermediate ADC values in comparison with normal brain. $D$, The APT image shows increased signal in both solid and necrotic portions of the tumor.
Net Reclassification Improvement to Evaluate the Added Value of APT Imaging to $\left[{ }^{18}\right.$ F] FDG-PET for the Diagnosis of High-Grade Glioma Table 4 summarizes the NRI results for each combination of the 2 parameters. When $\mathrm{APT}_{\text {mean }}$ was combined with the $\mathrm{T} / \mathrm{N}$ ratio, the continuous NRI was 0.64 (95\% CI, 0.036-1.24, $P=.04$ ) for diagnosis of high-grade glioma and 0.95 (95\% CI, 0.39-1.52; $P=.001$ ) for the diagnosis of glioblastoma.

\section{Validation}

Results of the internal validation are summarized in Tables 5 and 6.

\section{Additive Value of APT Imaging to DWI for Glioma Grading}

Table 7 summarizes the results of the AUCs for a combination of $\mathrm{ADC}_{\text {min }}$ and $\mathrm{APT}_{\text {mean }}$. In comparison with the AUC for $\mathrm{ADC}_{\text {min }}$ alone, some improvement was observed, though the difference did not reach statistical significance (grades III and IV versus grade II, $P=.36$; grade IV versus grades II and III, $P=.42)$. The continuous NRI was 0.48 (95\% CI, -0.13-1.09, $P=.12$ ) for the diagnosis of high-grade glioma and 1.14 (95\% CI, $0.58-1.71 ; P<.001)$ for diagnosis of glioblastoma when the $\mathrm{APT}_{\text {mean }}$ was combined with the $\mathrm{ADC}_{\text {min }}$. 


\section{DISCUSSION}

This study has 2 major findings. First, we demonstrated that $\mathrm{APT}_{\text {mean }}$ offered good diagnostic accuracy for high-grade glioma, comparable with that of other single imaging biomarkers such as $\mathrm{ADC}_{\text {min }}$ or the $\mathrm{T} / \mathrm{N}$ ratio from $\left[{ }^{18} \mathrm{~F}\right]$ FDG-PET. Second, our results also indicated that multiparametric analysis including APT and FDG-PET can improve the classification of gliomas of differing aggressiveness.

By focusing on amide protons, APT imaging has been used to visualize endogenous mobile proteins and peptides, and tissue $\mathrm{pH}$, without requiring administration of a contrast agent. ${ }^{9,28,29}$ The method involves a chemical exchange saturation transfer mechanism, with the signal changes observed being the result of a reduction in the bulk water signal intensity caused by chemical exchanges with magnetically labeled backbone amide protons on a resonance of around $+3.5 \mathrm{ppm}$ of that of free water.

Prior studies on APT imaging have been successfully applied to the assessment of human brain tumors. ${ }^{3,11-18}$ However, details

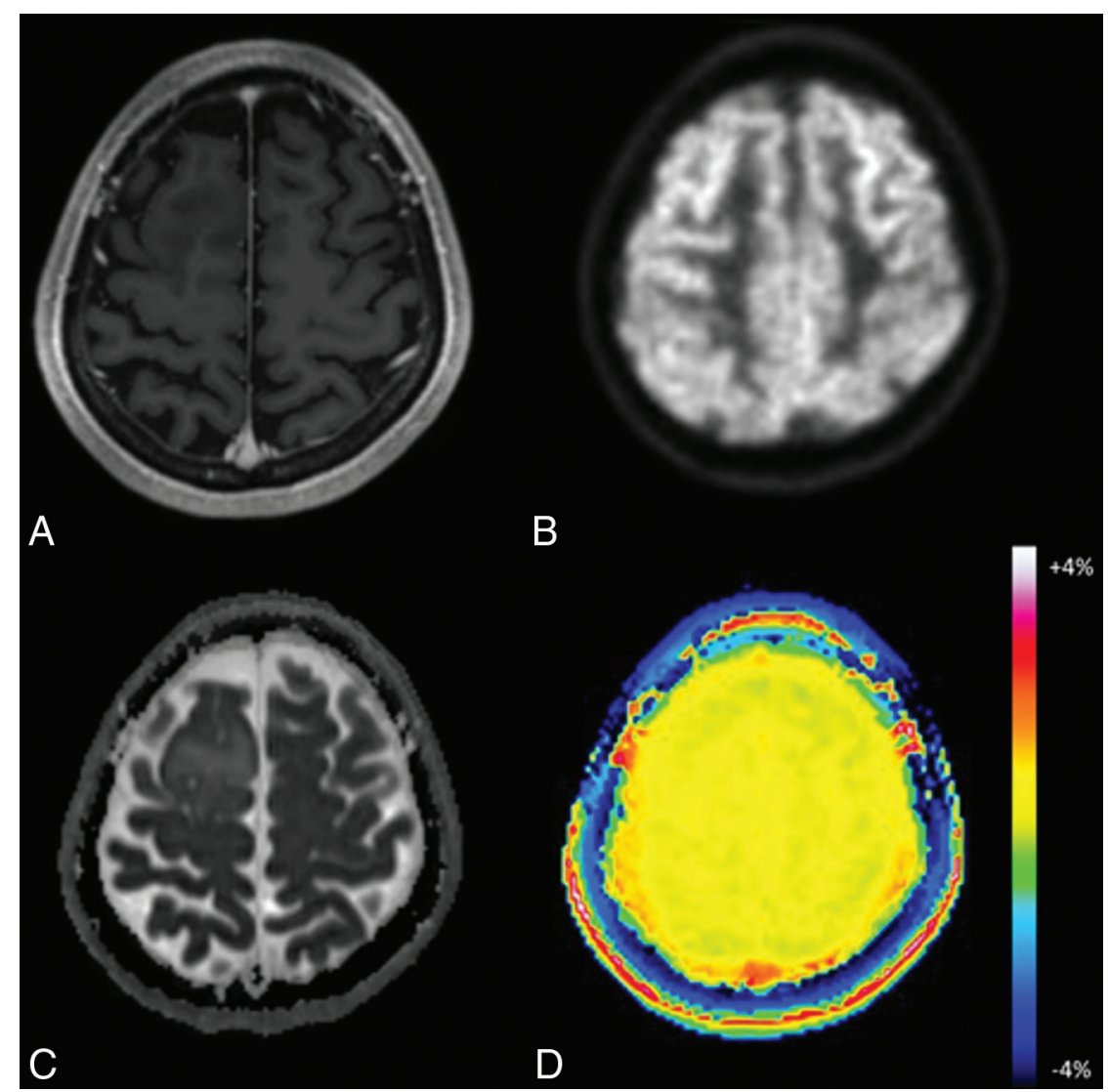

FIG 3. Oligodendroglioma in a 45-year-old woman. A, Axial MR imaging shows no contrastenhancing lesion in the right frontal lobe. B, FDG-PET demonstrates little uptake by the lesion compared with gray matter. $C$, On the ADC map, the tumor shows foci with no diffusion restriction within the tumor. $D$, The APT image shows no increased signal in this lesion. of the relationships between APT imaging and other clinical imaging parameters of malignancy have yet to be fully elucidated. This study confirmed that APT imaging can be used for grading glial tumors, with a diagnostic accuracy comparable with that of other imaging biomarkers derived from DWI and FDG-PET. Previous studies have shown the diagnostic accuracy of APT imaging to be comparable with DSC-PWI, ${ }^{13,16}$ and better than contrastenhanced T1-weighted imaging. ${ }^{22}$ As in previous studies, our results also demonstrated excellent interrater reproducibility in the measurement of APT. ${ }^{3,11-16}$ We believe that for the preoperative grading of brain tumors, APT can be considered an alternative approach to PET and other MR imaging methods such as DWI.

Multiparametric analysis including APT has the potential to improve the diagnostic accuracy in glioma grading. Several researchers have argued that multiparametric MR imaging methods have the potential to improve the diagnostic performance of preoperative glioma grading. ${ }^{30-32}$ Furthermore, Yoon et $\mathrm{al}^{1}$ reported that adding FDG-PET to multiparametric MR imaging, including DWI, PWI, and MR spectroscopy, can improve the diagnostic accuracy of glioma grading. However, few studies have examined glioma grading with multiparametric imaging that included APT imaging and PWI or DWI. ${ }^{10,16}$ To the best of our knowledge, our investigation represents the first study to show the utility of multiparametric analysis, including APT and PET, in the preoperative grading of gliomas.

To assess discrimination in the multiparametric logistic regression analysis, we applied 2 different statistical methods: ROC curve analysis and NRI. In ROC analysis, the AUC is commonly used to measure the discriminatory ability of a model to correctly classify subjects with or without a disease and has thus been a standard metric used to quantify improvement. However, this metric is known to have various limitations, including a lack of clinical relevance and difficulty in interpreting small-magnitude changes. ${ }^{16}$ We did not observe any significant gains to the AUCs with the addition of either $\mathrm{APT}_{\text {mean }}$ or $\mathrm{ADC}_{\text {min }}$ to the $\mathrm{T} / \mathrm{N}$ ratio. This was partly due to the relatively high diagnostic accuracy of each single method. As an alternative, NRI allows

Table 2: AUCs of each single parameter for predicting glioma grading

\begin{tabular}{|c|c|c|c|c|c|c|}
\hline & \multicolumn{2}{|c|}{$\begin{array}{c}\text { Grade II vs Grades } \\
\text { III and IV }\end{array}$} & \multirow[b]{2}{*}{ Optimal Cutoff } & \multicolumn{2}{|c|}{$\begin{array}{c}\text { Grades II and III vs } \\
\text { Grade IV }\end{array}$} & \multirow[b]{2}{*}{ Optimal Cutoff } \\
\hline & AUC & $95 \% \mathrm{Cl}$ & & AUC & $95 \% \mathrm{Cl}$ & \\
\hline $\mathrm{T} / \mathrm{N}$ & 0.84 & $0.72-0.97$ & 0.88 & 0.85 & $0.83-0.97$ & 1.03 \\
\hline $\mathrm{APT}_{\text {mean }}$ & 0.76 & $0.66-0.91$ & $1.26 \%$ & 0.86 & $0.76-0.97$ & $1.28 \%$ \\
\hline$A D C_{\min }$ & 0.78 & $0.62-0.90$ & $792.5 \times 10^{-6} \mathrm{~mm}^{2} / \mathrm{s}$ & 0.92 & $0.82-1.00$ & $788 \times 10^{-6} \mathrm{~mm}^{2} / \mathrm{s}$ \\
\hline
\end{tabular}


Table 3: AUCs of each combination of parameters for predicting glioma grading

\begin{tabular}{|c|c|c|c|c|c|c|}
\hline & \multicolumn{3}{|c|}{ Grade II vs Grades III and IV } & \multicolumn{3}{|c|}{ Grades II and III vs Grade IV } \\
\hline & AUC & $95 \% \mathrm{Cl}$ & $P$ Value & AUC & $95 \% \mathrm{Cl}$ & $P$ Value \\
\hline $\mathrm{T} / \mathrm{N}+\mathrm{APT}_{\text {mean }}$ & 0.85 & $0.73-0.97$ & .75 & 0.9 & $0.82-0.99$ & .24 \\
\hline $\mathrm{T} / \mathrm{N}+\mathrm{ADC}_{\min }$ & 0.86 & $0.76-0.97$ & .58 & 0.94 & $0.86-1.00$ & .19 \\
\hline $\mathrm{T} / \mathrm{N}$ & 0.84 & $0.72-0.97$ & NA & 0.85 & $0.73-0.98$ & NA \\
\hline
\end{tabular}

Note:-NA indicates not applicable.

Table 4: Continuous NRI results with the combination of 2 imaging parameters

\begin{tabular}{|c|c|c|c|c|c|c|}
\hline & \multicolumn{3}{|c|}{ Grade II vs Grades III and IV } & \multicolumn{3}{|c|}{ Grades II and III vs Grade IV } \\
\hline & NRI & $95 \% \mathrm{Cl}$ & $P$ Value & NRI & $95 \% \mathrm{Cl}$ & $P$ Value \\
\hline $\mathrm{T} / \mathrm{N}+\mathrm{APT}_{\text {mean }}$ & 0.64 & $0.036-1.24$ & .04 & 0.95 & $0.39-1.52$ & .001 \\
\hline $\mathrm{T} / \mathrm{N}+\mathrm{ADC}_{\min }$ & 0.43 & $-0.17-1.04$ & .16 & 1.36 & $0.79-1.92$ & $<.0001$ \\
\hline
\end{tabular}

Table 5: Validated logistic regression analysis results with the combination of 2 imaging parameters

\begin{tabular}{lcccccc}
\hline & \multicolumn{2}{c}{ Grade II vs Grades III and IV } & & \multicolumn{2}{c}{ Grade IV vs Grades II and III } \\
\cline { 2 - 3 } & AUC & $95 \% \mathrm{Cl}$ (Bias-Corrected) & & AUC & 95\% Cl (Bias-Corrected) \\
\hline $\mathrm{T} / \mathrm{N}+\mathrm{APT}_{\text {mean }}$ & 0.86 & $0.70-0.95$ & & 0.90 & $0.79-0.97$ \\
$\mathrm{~T} / \mathrm{N}+\mathrm{ADC}_{\text {min }}$ & 0.86 & $0.73-0.95$ & & 0.93 & $0.82-0.99$ \\
\hline
\end{tabular}

Table 6: Validated continuous NRI results with the combination of 2 imaging parameters

\begin{tabular}{lcccccc}
\hline & \multicolumn{2}{c}{ Grade II vs Grades III and IV } & & \multicolumn{2}{c}{ Grades II and III vs Grade IV } \\
\cline { 2 - 3 } & NRI & $95 \%$ Cl (Bias-Corrected) & & NRI & 95\% Cl (Bias-Corrected) \\
\hline $\mathrm{T} / \mathrm{N}+\mathrm{APT}_{\text {mean }}$ & 0.64 & $-0.14-1.26$ & & 0.9 & $0.29-1.46$ \\
$\mathrm{~T} / \mathrm{N}+\mathrm{ADC}_{\text {min }}$ & 0.49 & $-0.36-1.28$ & & 1.33 & $0.88-1.76$ \\
\hline
\end{tabular}

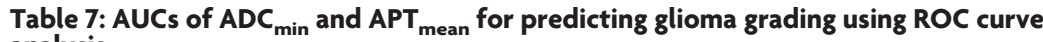
analysis

\begin{tabular}{lccccccc}
\hline & \multicolumn{3}{c}{ Grade II vs Grades III and IV } & & \multicolumn{3}{c}{ Grades II and III vs Grade IV } \\
\cline { 2 - 3 } & AUC & $95 \% C l$ & P Value & & AUC & $95 \% C l$ & P Value \\
\hline $\mathrm{ADC}_{\text {min }} \mathrm{APT}_{\text {mean }}$ & 0.82 & $0.71-0.94$ & .36 & & 0.94 & $0.86-1.00$ & .42 \\
$\mathrm{ADC}_{\text {min }}$ & 0.79 & $0.66-0.91$ & NA & & 0.92 & $0.82-1.00$ & NA \\
\hline
\end{tabular}

Note:-NA indicates not applicable.

quantification of the degree of correct reclassification. ${ }^{33,34}$ This measure is calculated as a change in the proportion of correct classifications minus incorrect ones, resulting from the new model in comparison with the former one. ${ }^{20}$ Using this approach, we demonstrated that the addition of $\mathrm{APT}_{\text {mean }}$ to the first model with a $\mathrm{T} / \mathrm{N}$ ratio achieved significant improvements, while the addition of $\mathrm{ADC}_{\text {min }}$ offered no significant improvement in the discrimination of high-grade gliomas. On the basis of our observations and prior reports that have shown moderate correlations between $\mathrm{APT}_{\text {asym }}$ values and histologic or other imaging biomarkers, we believe that APT shows potential as another useful and adjunctive biomarker of tumor aggressiveness in neuro-oncology.

We conducted further analysis to evaluate the additive value of APT imaging to DWI, and our results showed that a combination of APT and ADC did not significantly improve the primary outcome (ie, the diagnostic accuracy of high-grade glioma). This is different from a previous study that demonstrated a significant improvement. ${ }^{16}$ Differences in the acquisition parameters may explain the different findings of the 2 studies, but our results may indicate the importance of multitechnique imaging-based diagnosis.

Several limitations must be considered when interpreting the present results. This study was retrospective in design and included a relatively small cohort. We included approximately 20 patients with low-grade gliomas, which prevented us from conducting robust multivariate regression analyses with $>2$ variables. Second, we excluded patients with grade I glioma for 2 reasons: one being that adult cases with grade I glioma are relatively rare, with most patients with grade I glioma being diagnosed as having pilocytic astrocytoma in our hospital, in addition to being mostly younger than 18 years of age; and the second reason being that grade I glioma is known to be different from diffuse glioma in its pathologic, genetic, and even imaging characteristics. ${ }^{35,36}$ Third, the intervals between imaging and an operation were longer for low-grade gliomas than for high-grade gliomas. This difference was because elective operations were conducted for patients with suspected low-grade gliomas, with FDGPET being performed in the initial assessment of the tumor in our institution. FDG-PET was usually not repeated before the operation, due to the high cost and radiation exposure. We found no size increases on conventional MR imaging during this interval, so we do not consider this delay as likely to have affected the results, especially considering the relatively low proliferative activity of low-grade glioma.

Fourth, we analyzed only a single representative slice from the APT imaging. However, a previous study has shown that the difference of measurements on APT imaging has little effect on glioma grading. ${ }^{15}$ Fifth, we conducted this study using 2 scanners of the same type, with the same sequences and parameters. To our knowledge, there is no study assessing interscanner or intervendor differences in APT imaging. There may well be some interscanner differences, even between scanners of the same type; however, our results showed that APT imaging has additive value, even when different scanners of the same type are used. Finally, we did not examine the relationship between the imaging parameters and the molecular profiles of tumors. Several recent studies have clarified the importance of the molecular status of the tumor, including the mutation of genes such as IDH-1, ATRX, and TERT. ${ }^{37,38}$ We should conduct further studies to investigate the potential associations between imaging parameters and such molecular or genetic profiles of gliomas.

\section{CONCLUSIONS}

Our findings indicate that APT, DWI, and FDG-PET are useful for predicting the malignant grade of cerebral glioma. In combination with FDG-PET, APT showed the potential to improve the diagnostic performance in the identification of high-grade glioma. To investigate the accuracy of our results, external validation using larger samples should be conducted in future clinical studies. 


\section{ACKNOWLEDGMENTS}

We thank Karl Embleton, PhD, from Edanz Group (www.edanzediting.com/ac) for editing a draft of this article.

Disclosures: Tomohisa Okada-UNRELATED: Payment for Lectures Including Service on Speakers Bureaus: Siemens Healthcare Japan, Comments: honorarium for a talk; OTHER RELATIONSHIPS: I have a research collaboration with Siemens Healthcare, Japan. Benjamin Schmitt-UNRELATED: Employment: Siemens Healthcare, Comments: full-time employee. Kaori Togashi-RELATED: Grant: Ministry of Education, Culture, Sports, Science and Technology, Japan (No. 25120002), Comments: governmental research grant; OTHER RELATIONSHIPS: I am conducting collaborative work with Siemens Healthcare.

\section{REFERENCES}

1. Yoon JH, Kim JH, Kang WJ, et al. Grading of cerebral glioma with multiparametric MR imaging and 18F-FDG-PET: concordance and accuracy. Eur Radiol 2014;24:380-89 CrossRef Medline

2. Chung $\mathrm{C}$, Metser U, Ménard C. Advances in magnetic resonance imaging and positron emission tomography imaging for grading and molecular characterization of glioma. Semin Radiat Oncol 2015; 25:164-71 CrossRef Medline

3. Bai Y, Lin Y, Zhang W, et al. Noninvasive amide proton transfer magnetic resonance imaging in evaluating the grading and cellularity of gliomas. Oncotarget 2017;8:5834-42 CrossRef Medline

4. Higano S, Yun X, Kumabe T, et al. Malignant astrocytic tumors: clinical importance of apparent diffusion coefficient in prediction of grade and prognosis. Radiology 2006;241:839-46 CrossRef Medline

5. Murakami R, Hirai T, Sugahara T, et al. Grading astrocytic tumors by using apparent diffusion coefficient parameters: superiority of a one- versus two-parameter pilot method. Radiology 2009;251:838-45 CrossRef Medline

6. van Zijl PC, Yadav NN. Chemical exchange saturation transfer (CEST): what is in a name and what isn't? Magn Reson Med 2011;65: 927-48 CrossRef Medline

7. Zaiss M, Bachert P. Chemical exchange saturation transfer (CEST) and MR Z-spectroscopy in vivo: a review of theoretical approaches and methods. Phys Med Biol 2013;58:R221-69 CrossRef Medline

8. Vinogradov E, Sherry AD, Lenkinski RE. CEST: from basic principles to applications, challenges and opportunities. J Magn Reson 2013;229:155-72 CrossRef Medline

9. Zhou J, Payen JF, Wilson DA, et al. Using the amide proton signals of intracellular proteins and peptides to detect $\mathrm{pH}$ effects in MRI. Nat Med 2003;9:1085-90 CrossRef Medline

10. Zhou J, Lal B, Wilson DA, et al. Amide proton transfer (APT) contrast for imaging of brain tumors. Magn Reson Med 2003;50: 1120-26 CrossRef Medline

11. Togao O, Yoshiura T, Keupp J, et al. Amide proton transfer imaging of adult diffuse gliomas: correlation with histopathological grades. Neuro Oncol 2014;16:441-48 CrossRef Medline

12. Park JE, Kim HS, Park KJ, et al. Histogram analysis of amide proton transfer imaging to identify contrast-enhancing low-grade brain tumor that mimics high-grade tumor: increased accuracy of MR perfusion. Radiology 2015;277:151-61 CrossRef Medline

13. Togao O, Hiwatashi A, Yamashita K, et al. Grading diffuse gliomas without intense contrast enhancement by amide proton transfer MR imaging: comparisons with diffusion- and perfusion-weighted imaging. Eur Radiol 2017;27:578-88 CrossRef Medline

14. Park JE, Kim HS, Park KJ, et al. Pre- and posttreatment glioma: comparison of amide proton transfer imaging with MR spectroscopy for biomarkers of tumor proliferation. Radiology 2016;278: 514-23 CrossRef Medline

15. Sakata A, Okada T, Yamamoto A, et al. Grading glial tumors with amide proton transfer MR imaging: different analytical approaches. J Neurooncol 2015;122:339-48 CrossRef Medline

16. Choi YS, Ahn SS, Lee SK, et al. Amide proton transfer imaging to discriminate between low- and high-grade gliomas: added value to apparent diffusion coefficient and relative cerebral blood volume. Eur Radiol 2017;27:3181-89 CrossRef Medline

17. Ma B, Blakeley JO, Hong X, et al. Applying amide proton transferweighted MRI to distinguish pseudoprogression from true progression in malignant gliomas. J Magn Reson Imaging 2016;44: 456-62 CrossRef Medline

18. Park KJ, Kim HS, Park JE, et al. Added value of amide proton transfer imaging to conventional and perfusion MR imaging for evaluating the treatment response of newly diagnosed glioblastoma. Eur Radiol 2016;26:4390-403 CrossRef Medline

19. Sagiyama $\mathrm{K}$, Mashimo T, Togao $\mathrm{O}$, et al. In vivo chemical exchange saturation transfer imaging allows early detection of a therapeutic response in glioblastoma. Proc Natl Acad Sci U S A 2014;111: 4542-47 CrossRef Medline

20. Pencina MJ, D’Agostino RB Sr, D’Agostino RB Jr, et al. Evaluating the added predictive ability of a new marker: from area under the ROC curve to reclassification and beyond. Stat Med 2008;27: 157-72; discussion 207-12 CrossRef Medline

21. Louis DN, Ohgaki H, Wiestler OD, et al. The 2007 WHO classification of tumours of the central nervous system. Acta Neuropathol 2007;114:97-109 CrossRef Medline

22. Sakata A, Fushimi Y, Okada T, et al. Diagnostic performance between contrast enhancement, proton MR spectroscopy, and amide proton transfer imaging in patients with brain tumors. J Magn Reson Imaging 2017;46:732-39 CrossRef Medline

23. Schmitt B, Zaiss M, Zhou J, et al. Optimization of pulse train presaturation for CEST imaging in clinical scanners. Magn Reson Med 2011;65:1620-29 CrossRef Medline

24. Yamashita K, Yoshiura T, Hiwatashi A, et al. Differentiating primary CNS lymphoma from glioblastoma multiforme: assessment using arterial spin labeling, diffusion-weighted imaging, and ${ }^{18} \mathrm{~F}$-fluorodeoxyglucose positron emission tomography. Neuroradiology 2013; 55:135-43 CrossRef Medline

25. Manabe O, Hattori N, Yamaguchi S, et al. Oligodendroglial component complicates the prediction of tumour grading with metabolic imaging. Eur J Nucl Med Mol Imaging 2015;42:896-904 CrossRef Medline

26. Landis JR, Koch GG. The measurement of observer agreement for categorical data. Biometrics 1977;33:159-74 CrossRef Medline

27. DeLong ER, DeLong DM, Clarke-Pearson DL. Comparing the areas under two or more correlated receiver operating characteristic curves: a nonparametric approach. Biometrics 1988;44: 837-45 CrossRef Medline

28. Schmidt H, Schwenzer NF, Gatidis S, et al. Systematic evaluation of amide proton chemical exchange saturation transfer at $3 \mathrm{~T}$ : effects of protein concentration, $\mathrm{pH}$, and acquisition parameters. Invest Radiol 2016;51:635-46 CrossRef Medline

29. Sun PZ, Wang E, Cheung JS. Imaging acute ischemic tissue acidosis with $\mathrm{pH}$-sensitive endogenous amide proton transfer (APT) MRI: correction of tissue relaxation and concomitant RF irradiation effects toward mapping quantitative cerebral tissue $\mathrm{pH}$. Neuroimage 2012;60:1-6 CrossRef Medline

30. Fudaba H, Shimomura $\mathrm{T}, \mathrm{Abe} \mathrm{T}$, et al. Comparison of multiple parameters obtained on $3 \mathrm{~T}$ pulsed arterial spin-labeling, diffusion tensor imaging, and MRS and the Ki-67 labeling index in evaluating glioma grading. AJNR Am J Neuroradiol 2014;35:2091-98 CrossRef Medline

31. Server A, Kulle B, Gadmar ØB, et al. Measurements of diagnostic examination performance using quantitative apparent diffusion coefficient and proton MR spectroscopic imaging in the preoperative evaluation of tumor grade in cerebral gliomas. Eur J Radiol 2011;80:462-70 CrossRef Medline

32. Nguyen TB, Cron GO, Perdrizet K, et al. Comparison of the diagnostic accuracy of DSC- and dynamic contrast-enhanced MRI in the preoperative grading of astrocytomas. AJNR Am J Neuroradiol 2015; 36:2017-22 CrossRef Medline

33. Halligan S, Altman DG, Mallett S. Disadvantages of using the area under the receiver operating characteristic curve to assess imaging 
tests: a discussion and proposal for an alternative approach. Eur Radiol 2015;25:932-39 CrossRef Medline

34. Rutjes A, Reitsma J, Coomarasamy A, et al. Evaluation of diagnostic tests when there is no gold standard: a review of methods. Health Technol Assess 2007;11:iii, ix-51 Medline

35. Collins VP, Jones DT, Giannini C. Pilocytic astrocytoma: pathology, molecular mechanisms and markers. Acta Neuropathol 2015;129: 775-88 CrossRef Medline

36. de Fatima Vasco Aragao M, Law M, Batista de Almeida D, et al. Com- parison of perfusion, diffusion, and MR spectroscopy between lowgrade enhancing pilocytic astrocytomas and high-grade astrocytomas. AJNR Am J Neuroradiol 2014;35:1495-502 CrossRef Medline

37. Eckel-Passow JE, Lachance DH, Molinaro AM, et al. Glioma groups based on 1p/19q, IDH, and TERT promoter mutations in tumors. N Engl J Med 2015;372:2499-508 CrossRef Medline

38. Koschmann C, Calinescu AA, Nunez FJ, et al. ATRX loss promotes tumor growth and impairs nonhomologous end joining DNA repair in glioma. Sci Transl Med 2016;8:328ra28 CrossRef Medline 